\title{
BIOOXYDATION DE MINÉRAUX SULFURÉS ET DISSOLUTION DE MÉTAUX PAR LA BACTÉRIE ACIDOPHILE : THIOBACILLUS FERROOXIDANS
}

\author{
Mireille Bruschi, Christine Cavazza, Marie Thérèse Giudici-Orticoni* \\ Bionergétique et Ingénierie des Protéines CNRS - IFR I
}

\begin{abstract}
L'extraction industrielle de métaux à partir de minerais par biolixiviation met à profit la faculté d'oxyder le fer de Thiobacillus ferrooxidans. L'oxydation du fer fait intervenir une chaîne de transfert d'électrons localisée dans le périplasme de la cellule. La caractérisation des protéines d'oxydoréduction impliquées, la détermination de leur rôle physiologique et les techniques du génie génétique pourront permettre d'améliorer le processus de biolixiviation.
\end{abstract}

The commercial extraction of metals from ores by microbial leaching turns to account the iron oxidation capabilities of Thiobacillus ferrooxidans. The iron oxidation involves an electron transfer chain localized in the periplasmic space of the cell. The characterization of the redox proteins involved the determination of their physiological role, together with genetic engineering programs, could have implications to increase the leaching capacity of these bacteria.

Le respect de l'environnement apparaît maintenant comme une préoccupation essentielle dans les enjeux économiques et sociaux d'une société. Cette prise de conscience grandissante se traduit notamment par la volonté de développer les biotechnologies et les bio-industries. Parmi celles-ci, s'inscrit la biolixiviation dont le devenir s'insère dans un triple enjeu :

- la gestion des ressources naturelles,

- la protection de l'environnement,

- la maîtrise de l'énergie.

La biolixiviation peut être définie comme un procédé biotechnologique industriel qui permet de valoriser certains types de minerais, soit pauvres, soit difficiles à traiter (or, cuivre, uranium...). Elle met à profit la capacité de certains micro-organismes, d'attaquer des composés insolubles et de conduire à la mise en solution des éléments qui les composent.

Le minerai, mis en tas, est arrosé par une solution lixiviante (composée de micro-organismes) et le métal est récupéré après percolation de la solution.

Comme pour d'autres procédés biotechnologiques, nos ancêtres en avaient fait l'expérience. Des vestiges archéo- logiques montrent que, mille ans avant $\mathrm{JC}$, les peuples du pourtour méditerranéen savaient récupérer le cuivre présent dans les eaux de drainage des mines. Dès le XVIII siècle, la lixiviation était également utilisée en Espagne, pour extraire le cuivre dans les mines du Rio Tinto.

Actuellement, de nombreux groupes industriels, aussi bien aux États-Unis qu'en Amérique du Sud et en Europe, s'impliquent financièrement dans les enjeux économiques et scientifiques de la biolixiviation. Plus de $10 \%$ du cuivre produit mondialement est obtenu par ce procédé tandis que des essais à grande échelle sont en cours, concernant la récupération de cobalt, d'or ou d'argent. Les minerais sont prétraités par les bactéries, de manière à débarrasser les particules métalliques de leur gangue sulfurée qui les rend inexploitables par cyanuration directe.

Cette mise en œuvre est peu coûteuse car elle nécessite un apport énergétique très réduit par rapport aux exploitations classiques. Elle permet une baisse de prix de revient du métal traité et une économie d'énergie. Cette technique s'inscrit également dans une politique de protection de l'environnement. En effet, elle évite l'émission, dans l'atmosphère, de composés sulfurés et toxiques, actuellement produits dans les exploitations classiques. De plus, elle intervient directement dans les procédés de dépollution (élimination des composés sulfurés dans les charbons et les pétroles, récupération de métaux lourds dans les déchets industriels...).

Toutefois, ces processus sont aussi à l'origine des phénomènes d'acidification des eaux et des sols.

\section{UNE BACTÉRIE ACIDOPHILE, THIOBACILLUS FERROOXIDANS}

Peu de bactéries sont capables de vivre dans des environnements adverses tels que des $\mathrm{pH}$, des températures extrêmes ou de très fortes salinités. Certaines eubactéries acidophiles sont très bien adaptées à la croissance à des $\mathrm{pH}$ très bas. L'une d'entre elles, chimiolithotrophe stricte et acidophile, Thiobacillus ferrooxidans, a développé l'une des curiosités bioénergétiques de la nature : la vie en milieu acide, aux dépens de l'oxydation du fer ferreux et de sulfures, 
en présence de l'oxygène de l'air. Le fer ferrique, oxydant puissant en milieu acide, conduit à la dissolution des sulfures et des métaux présents dans les roches et minerais. Le fer ferreux, régénéré par la dissolution des minerais, est, en permanence, disponible pour la croissance bactérienne. Malgré son intérêt industriel, nos connaissances générales sur la physiologie, la génétique et la biochimie de cet organisme sont très réduites. Plusieurs raisons expliquent ce fait : le très bas $\mathrm{pH}$ de croissance (I,6 à 2), le temps de génération de plusieurs heures, la toxicité des produits organiques, qui entraîne une grande difficulté de culture sur gélose à très bas $\mathrm{pH}$, celle-ci étant hydrolysée en donnant du galactose. De ce fait, peu de progrès ont été faits pour améliorer l'utilisation de Thiobacillus ferrooxidans pour la biolixiviation et la récupération de métaux d'intérêt, à partir de minerais pauvres ou de sous-produits industriels contaminés par des métaux toxiques. Le but des études entreprises, en collaboration entre plusieurs laboratoires, est de mieux comprendre les stratégies moléculaires mises en jeu par cette bactérie pour s'adapter aux conditions extrêmes de croissance, de façon à améliorer ses capacités biolixiviantes dans le contrôle des résidus miniers ou de la dépollution. Les différents thèmes abordés sont :
- l'adhésion des bactéries sur les surfaces minérales (programme du groupe de J. Berthelin - Centre de pédologie biologique - Nancy) ;

- la mise en évidence des chaînes de transfert d'électrons entre le fer ferreux et l'oxygène ou le thiosulfate (groupe $M$. Bruschi - Bioénergétique et ingénierie des protéines. Marseille) ;

- l'étude de la chaîne de transfert inverse d'électrons nécessaire à la fixation du $\mathrm{CO}_{2}$ pour la croissance de la bactérie (groupe D. Lemesle-Meunier - Bioénergétique et ingénierie des protéines. - Marseille) ;

- et l'étude génétique de la bactérie (groupe M. Chippaux

- Laboratoire de Chimie Bactérienne - Marseille).

\section{ÉTUDE DES CHAÎNES DE TRANSFERT D'ÉLECTRONS LIÉS À L'OXYDATION DU FER CHEZ THIOBACILLUS FERROOXIDANS}

Les difficultés de mise en œuvre d'un tel programme impliquent d'abord la maîtrise et la production en masse des bactéries. Aussi, la première partie de ce travail a été consacrée à la conception et à la construction d'un fermenteur de 250 litres, entièrement en plastique pour éviter la corrosion,

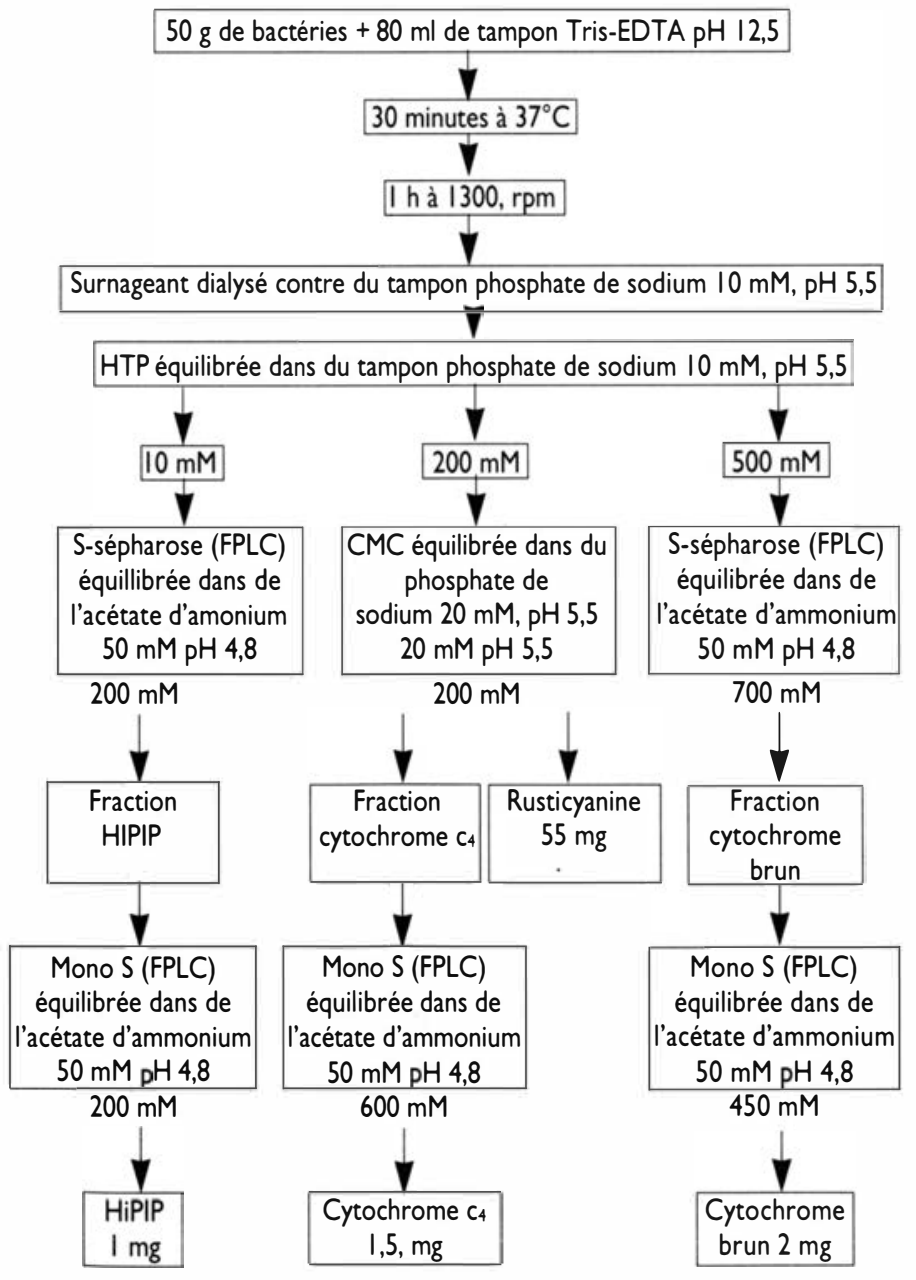

Figure I : Purification des protéines d'oxydoréduction de hiobacillus ferrooxidans par extraction périplasmique 
et spécialement adapté à la culture de Thiobacillus ferrooxidans à $\mathrm{pH}$ acide $(2,0)$. Le rendement de croissance de cette bactérie extrèmophile est particulièrement faible puisque seulement $15 \mathrm{~g}$ de cellules poids humide sont obtenus en moyenne à partir de $250 \mathrm{I}$ de culture. Les cultures étant effectuées environ 30 semaines par an, la quantité de cellules récoltées est d'environ $450 \mathrm{~g}$ par an.

\section{Étude biochimique des protéines solubles de transfert d'électrons impliquées dans l'oxydation du fer et du soufre}

Le but du programme de recherche est d'analyser les relations structure/fonction des protéines périplasmiques impliquées dans l'oxydation du fer et d'établir la séquence des partenaires d'oxydoréduction dans la chaîne respiratoire.

Deux chaînes de transfert d'électrons sont responsables de la production d'énergie nécessaire à la croissance des cellules, selon que la bactérie croît aux dépens du fer ferreux (Fell) ou du soufre. Dans la première, les électrons sont transférés du Fell jusqu'à l'oxygène ; dans la seconde, ils le sont depuis les composés sulfurés réduits du minéral à l'oxygène.

Notre démarche est donc, dans un premier temps, de purifier, par extraction périplasmique, les protéines d'oxydoréduction, présentes dans le périplasme de Thiobacillus ferrooxidans lorsque la bactérie est cultivée sur sulfate de fer (figure I) ; puis, de caractériser ces protéines. Ainsi, quatre protéines d'oxydoréduction périplasmiques pouvant être impliquées dans la chaîne de transfert d'électron entre le fer ferreux et l'oxygène ont été mises en évidence :

- la rusticyanine, qui représente $5 \%$ des protéines totales ; Cette protéine possède une masse moléculaire de 16500 Da et appartient à la famille des protéines à cuivre de type I. Cependant, sa présence dans une bactérie acidophile dont le donneur d'électrons est un substrat à potentiel d'oxydoréduction élevé lui confère deux particularités atypiques : elle présente une grande stabilité pour des valeurs de $\mathrm{pH}$ très basses et elle possède un potentiel d'oxydoréduction très élevé : + 680 mV à pH 3,2 (Nunzi et al., 1993).

- une protéine HiPIP (high potential iron-sulfur protein), similaire à la protéine Iro, dont le gène a été cloné et séquencé chez une autre souche de Thiobacillus ferrooxidans est purifiée sous sa forme tétramérique (chaque sous-unité de $6000 \mathrm{Da}$ contenant un centre 4Fe/4S). Le spectre RPE, ainsi que la valeur élevé de son potentiel d'oxydoréduction $(+490$ $\mathrm{mV}$ à $\mathrm{pH} 2$ ) sont typiques d'une protéine HiPIP (Cavazza et al., 1995).

- un cytochrome $\mathrm{c}_{4}$ a été isolé, pour la première fois, chez Thiobacillus ferrooxidans. Ce cytochrome possède une masse moléculaire de $21 \mathrm{kDa}$ et contient deux hèmes de type $\mathrm{c}$. L'étude par RPE met en évidence la présence des deux hèmes, qui présentent des valeurs de potentiels d'oxydoréduction distinctes $(+385$ et $+480 \mathrm{mV}$ à pH 3) (Cavazza et al., sous presse).

- un nouveau cytochrome $\mathrm{c}$ atypique a également été purifié. Cette protéine monomérique possède une masse molé- culaire de $13 \mathrm{kDa}$ et le dosage en fer met en évidence la présence d'un seul hème par molécule. Son spectre UV-visible montre la présence d'une bande entre 620 et $640 \mathrm{~nm}$ dans l'état oxydé, typique d'un cytochrome de type c'. Cependant, la séquence $\mathrm{N}$-terminale possède un site de fixation pour un hème $\mathrm{c}$, alors que l'hème est localisé dans la région $\mathrm{C}$-terminale chez les cytochromes c' (Cavazza et Bruschi, 1995).

\section{Étude cinétique permettant la reconstitu- tion des chaînes de transfert d'oxydation du fer}

Il existe à l'heure actuelle trois modèles décrivant le transfert d'électrons du fer ferreux à la cytochrome oxydase, accepteur terminal, qui font intervenir différentes protéines periplasmiques d'oxydoréduction (figure 2).

En dehors du modèle proposé par Ingledew (figure 2 c), les deux autres hypothèses excluent soit la participation de la rusticyanine pour Yamanaka et al. soit celle de l'HiPIP pour Blake et al. (Blake et Shute, 1994).

Nous avons étudié la cinétique de réduction de la rusticya-

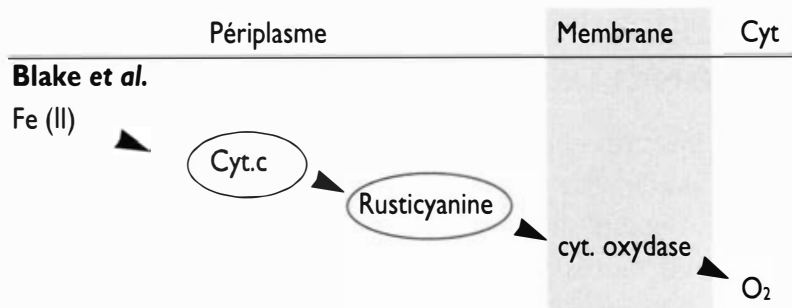

Yamanaka et al.

$\mathrm{Fe}$ (II)
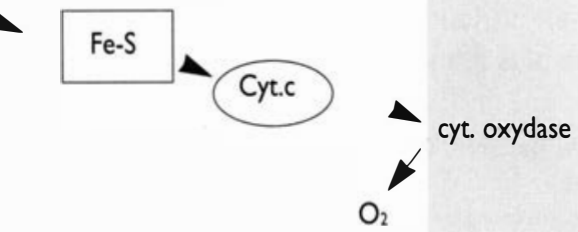

Ingledew et al.

$\mathrm{Fe}$ (II)

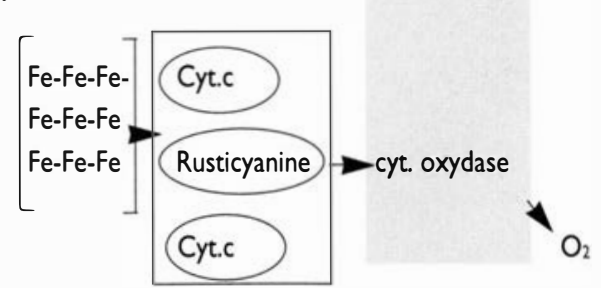

Figure 2 : Plusieurs schémas hypothétiques entre le fer ferreux et l'oxygène moléculaire chez Thiobacillus ferrooxidans sont proposés

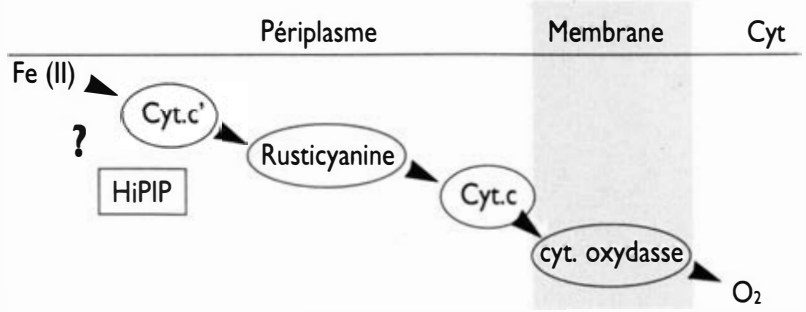

Figure 3 : Schéma hypothétique de la chaîne respiratoire de Thiobacillus ferrooxidans (cette étude) 
nine par le sulfate de fer. La vitesse (turn-over) de la réaction qui est de $0,014 \mathrm{~s}^{-1}$ montre que la rusticyanine n'est vraisemblablement pas le premier accepteur d'électron provenant du fer ferreux puisque la réduction de la rusticyanine, sur cellules entières (Blake et Shute, 1994), a une vitesse 200 fois plus grande. Les études réalisées avec le cytochrome $C_{4}$ mettent en évidence qu'il n'est pas réduit pas le sulfate de fer. En revanche, l'addition de rusticyanine dans le milieu réactionnel permet la réduction du cytochrome. II existe donc un transfert d'électron partant du fer et aboutissant au cytochrome $c_{4}$ via la rusticyanine.

L'utilisation d'une nouvelle technologie basée sur le phénomène de résonance plasmonique de surface (Biacore Pharmacia) nous a permis de montrer l'existence d'une interaction spécifique entre ces deux protéines $\left(\mathrm{Kd}=2 \cdot 10^{-7} \mathrm{M}\right)$. L'existence d'un transfert d'électron et d'une interaction spécifique entre ces deux protéines associée au fait que les cytochromes de type $c_{4}$ interagissent très souvent avec la membrane nous a conduit à proposer un modèle où la rusticyanine recevrait ses électrons du fer via l'HiPIP ou le cytochrome atypique et les transférerait au cytochrome $c_{4}$ qui réduirait à son tour la cytochrome oxydase (figure 3 ).

\section{CONCLUSION}

L'identification et la caractérisation de protéines d'oxydoréduction directement impliquées dans l'oxydation du fer et dans le métabolisme énergétique de Thiobacillus ferrooxidans sont importantes pour la mise en solution de métaux d'intérêt industriel ou, au contraire, toxiques. Cette étude peut permettre également, par ingénierie génétique, de modifier ces gènes ou leur régulation pour orienter les bactéries vers une plus grande capacité biolixiviante.

* Mireille Bruschi, Christine Cavazza, Marie Thérèse GiudiciOrticoni

Bionergétique et Ingénierie des Protéines CNRS - IFR I - 3I, chemin Joseph Aiguier - 13402 Marseille cedex 20

\section{Bibliographie}

I. Blake, R.C. II and Shute, E.A. (1994). Respiratory enzymes of Thiobacillus ferrooxidans. kinetic properties of an acidstable iron : rusticyanin oxidoreductase. Biochemistry 33, 9220-9228.

2. Cavazza, C. and Bruschi, M. (1995). Iron oxidation by Thiobacillus ferrooxidans : characterisation of two electron transfer proteins. Biohydrometallurgical Processing, Vol. II. Jerz, Vargas, Toledo and Wiertz Eds, pp 97-107.

3. Cavazza, C., Giudici-Orticoni, M.T. Nitschke, W., Appia, C., Bonnefoy V. and Bruschi, M. Characterisation of a new cytochrome $c_{4}$ from Thiobacillus ferrooxidans. Eur. J. of Biochem., sous presse.

4. Cavazza, C., Guigliarelli, B., Bertrand, P. and Bruschi, M. (1995). Biochemical and EPR characterization of a high potential iron-sulfur protein in Thiobacillus ferrooxidans. FEMS Microbiol. Lett. 130. 193-200.

5. Nunzi, F., Woudstra, M., Campése, D., Bonicel, J., Morin, D. and Bruschi, M. (1993). Amino acid sequence of rusticyanin from Thiobacillus ferrooxidans and its comparison with other blue copper proteins. Biochim. Biophys. Acta I 162, 28- 